\title{
SN contributions to GRB lightcurves $\left(^{*}\right)$
}

\author{
E. J. A. Meurs and M. C. A. Rebelo \\ Dunsink Observatory, Dublin, Ireland
}

(ricevuto il 23 Maggio 2005; pubblicato online il 12 Ottobre 2005)

\begin{abstract}
Summary. - Several of the nearer GRB afterglows (up to $z \sim 1$ ) show bumps in their lightcurves that have been interpreted as contributions from associated SNae. The bumps are customarily modelled like the type-Ic SN 1998bw, but we investigate here, for several low- $z$ GRBs, whether other SN types might offer alternatives. While several SN types are ruled out, or are unlikely, a type "II-bl" could also explain the observations.
\end{abstract}

PACS 98.70.Rz $-\gamma$-ray source, $\gamma$-ray burst.

PACS 01.30.Cc - Conference proceedings.

\section{1. - Introduction}

Bumps superposed on the decay lightcurves of Gamma Ray Burst afterglows have been interpreted as evidence for Supernovae associated with the GRB event [1]. After the notable coincidence between GRB980425 and SN1998bw, the optical lightcurve of this type Ic SN has become a standard choice as SN lightcurve to be used for lightcurve modelling (e.g. $[1,2,7]$ ). We have taken a more general look at the appropriateness of this SN lightcurve template. We consider specifically two relevant questions: would any other SN type provide an acceptable fit to the afterglow lightcurve bumps, and can some or most SN types clearly be excluded.

\section{2. - SN additions to GRB lightcurves}

Generally, GRB afterglow lightcurves exhibit power law decays [5] that are documented most comprehensively in the $\mathrm{R}$ band. The superposed bumps that are attributed to SNae appear after two to four weeks, when the SNae reach maximum optical brightness. It has become clear that SN contributions are preferentially seen at low redshifts $(z \lesssim 1$; see also [3]) and stand out better when superposed on steep afterglow decays.

$\left(^{*}\right)$ Paper presented at the "4th Workshop on Gamma-Ray Burst in the Afterglow Era", Rome, October 18-22, 2004.

(C) Società Italiana di Fisica 
TABLE I. - Possible SN types for three nearby GRBs.

\begin{tabular}{lcccl}
\hline GRB name & $z$ & $\alpha{\text { (our fit })^{a}}$ & $\alpha$ (lit.) $^{a}$ & Possible SN types \\
\hline 011121 & 0.362 & 1.71 & 1.72 & Ia,Ic,98bw; II-bl,(-bp),(-pec) \\
990712 & 0.434 & 0.98 & 1.03 & (Ib),94I,(II-L) \\
970228 & 0.695 & 1.55 & 1.58 & (Ia),Ic,98bw; II(-bl),-bp \\
\hline
\end{tabular}

$\left({ }^{a}\right)$ The quoted $\alpha$ values refer to the optical decay slopes.

Besides type-Ic Supernovae - epitomized by SN1998bw - there are the "conventional" types Ia, Ib, II-P[lateau] and II-L[inear]. A recent re-analysis of SN type-II lightcurves by Patat et al. [6] led to a somewhat different emphasis, with distinction between bright, regular and faint types II, with possibly further qualifications such as plateau, linear or peculiar.

Thus, we have generated composite lightcurves for three nearby $(z<0.8)$ GRBs, adding lightcurves for all the SN types cited above to the observed power law decays. We restrict ourselves to $R$-magnitude data, with any host galaxy contributions subtracted. The necessary corrections that account for the redshift of the objects have been made; apart from luminosity distance and time dilation, the $K$-correction and cosmological dimming were taken from Nugent et al. [4].

\section{3. - Results}

Some information on these three GRBs (GRB011121, GRB990712 and GRB970228) is presented in Table I. Figures 1 and 2 show, as an example, the composite lightcurves

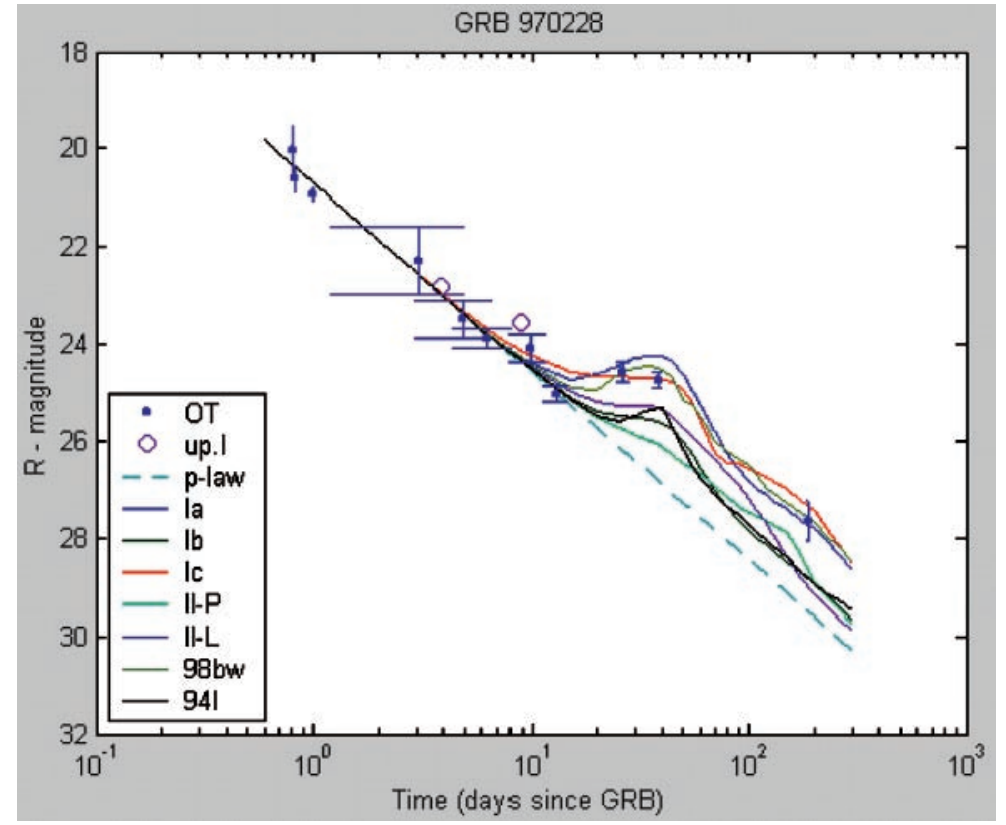

Fig. 1. - Lightcurves for conventional SN classification added to GRB970228 power law decay. 


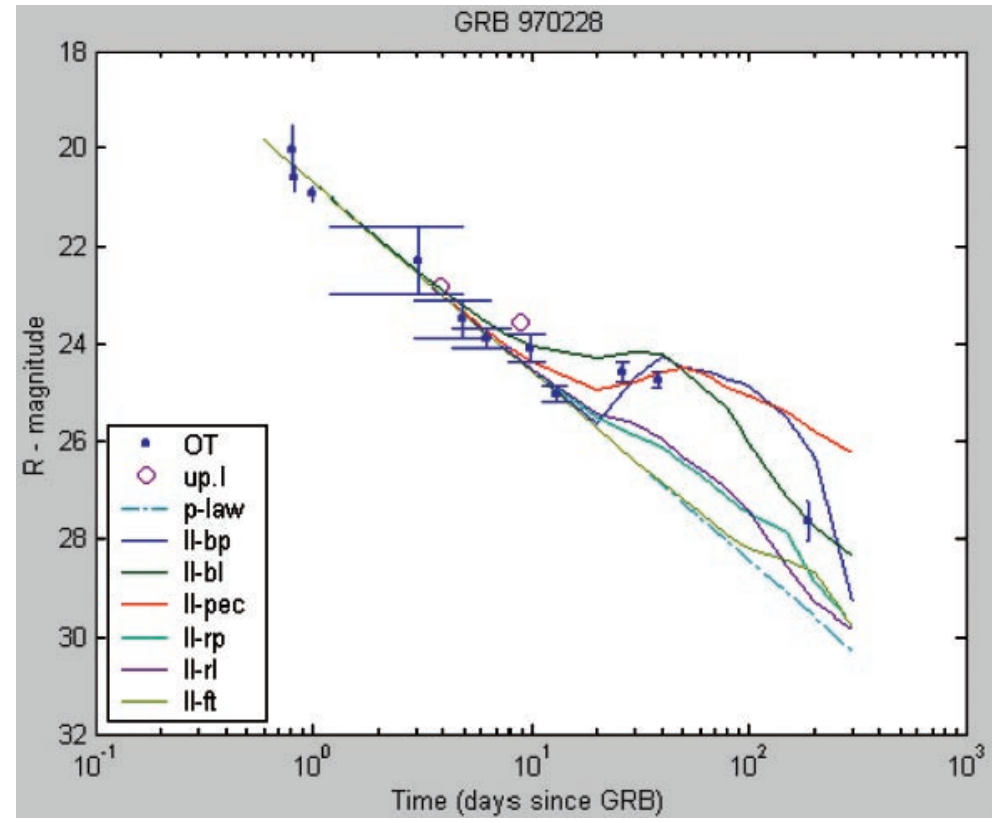

Fig. 2. - SN lightcurves for newer type II subdivision added to GRB970228 power law decay.

for GRB970228. There are two collections of SN types: fig. 1 is for the "conventional" SN classification (including a general type-Ic lightcurve, as well as specifically SN1998bw and SN1994I), while fig. 2 refers to the newer type-II subdivision in Patat et al. [6]. For each SN type we have assessed the success of its lightcurve addition quantitatively, by means of the value of the mean Observed-Calculated difference. The results of this assessment are quoted in the final column of table I. The original demonstration of a SN bump was for GRB980326; this one is not included here because its redshift has not been accurately determined (in fact, the $z$-dependent composite curves provided an estimate of $z$ ).

\section{4. - Conclusions}

The three GRB lightcurve models presented here constitute of course a very small sample. A further complication is that GRB990712 is suspected to have a SN contribution that is sub-luminous; the best match is provided by SN1994I (a type-Ic SN).

Nevertheless, the indication is that besides SN1998bw and type Ic generally, two of the type II subdivisions as pursued by [6] could provide suitable SN lightcurve matches: II-bl (bright-linear) and - although to a lesser degree - II-bp (bright-plateau). Like type-Ic SNae, these are thought to refer to massive stars. It is noted that, from the more conventional types, Type Ia is also reasonably successful.

The majority of the other SN types can be clearly excluded (Ib, II-L, II-P; II regularlinear/plateau, II faint).

The restriction to $R$-magnitudes is a consequence of the predominant emphasis of these magnitudes in GRB afterglow follow-up efforts. The composite lightcurves produced in the present work cannot be applied as such to other filterbands, due to differences in $K$-correction and to colour evolution of the SN lightcurves. 
For future extension of this work, an interesting point will be to examine whether the apparent absence of any SN contribution can be translated into an actual constraint on distance.

\section{REFERENCES}

[1] Bloom J. S., et al., Nature, 401 (1999) 453.

[2] Bloom J. S., et al., ApJ, 572 (2002) L45.

[3] Meurs E. J. A. and Norci L., ASPC, 312 (2004) 291.

[4] Nugent P., Kim A. and Perlmutter S., PASP, 114 (2002) 803.

[5] Van Paradijs J., Kouveliotou C. and Wijers R. A. M. J., ARAA, 38 (2000) 379.

[6] Patat F., Barbon R., Cappellaro E. and Turatto M., AA, 282 (1994) 731.

[7] Reichart D. E., ApJ, 521 (1999) L111. 